\title{
Interleukin 6 expression in coronary circulation after coronary angioplasty as a risk factor for restenosis
}

Y Hojo, U Ikeda, T Katsuki, O Mizuno, H Fukazawa, K Kurosaki, H Fujikawa, K Shimada

\begin{abstract}
Objective-To investigate changes in cytokine expression in the coronary circulation induced by percutaneous transluminal coronary angioplasty (PTCA).

Methods-The study involved 32 patients with ischaemic heart disease who underwent elective PTCA for isolated stenotic lesions of the left coronary artery. Ten patients had plain old balloon angioplasty, 10 had percutaneous transluminal rotational atherectomy, and 12 had stent implantation. Blood samples were drawn from the coronary sinus before and immediately after PTCA. Plasma concentrations of interleukin 6 (IL-6), platelet derived growth factor (PDGF), monocyte chemoattractant protein 1 (MCP-1), and macrophage coronary stimulating factor (M-CSF) were measured. The patients were scheduled for follow up angiography six months after PTCA. Late loss index was calculated using quantitative coronary angiography.

Results-IL-6 concentrations in coronary sinus blood increased immediately after PTCA $(\mathrm{p}<0.001$ ), but there was no change in PDGF, MCP-1, or M-CSF. There was a positive correlation between changes in IL-6 concentrations immediately after PTCA and late loss index six months after PTCA $(r=0.73, \mathrm{p}<0.001)$. IL-6 concentrations in coronary sinus blood were higher in patients with late restenosis than in those without restenosis $(p<0.001)$.

Conclusions-PTCA induces IL-6 production in the coronary circulation. This may induce subsequent inflammatory responses in injured vessels and play an important role in late restenosis after PTCA.

(Heart 2000;84:83-87)
\end{abstract}

Keywords: inflammation; cytokine; restenosis

Cytokines play a major role in cardiovascular diseases by inducing inflammation, cell proliferation, and apoptosis. It is well known that activated macrophages are one of the main sources of inflammatory cytokines. Previous studies have shown that the cells found in the vascular wall, such as vascular smooth muscle cells and endothelial cells, also produce various kinds of cytokines which are involved in cardiovascular diseases. ${ }^{1-3}$ It has been reported that the expression of proinflammatory cytokines such as interleukin 6 (IL-6) and IL-8 is increased in acute coronary syndromes. ${ }^{4-8}$ Enhanced expression of these cytokines is believed to be involved in acute and chronic complications of percutaneous transluminal coronary angioplasty (PTCA) by causing release of vasoactive substances, generating thrombus, and inducing other growth factors, thereby establishing a positive autocrine and paracrine feedback loop-a "cascade mechanism." The results of several studies have suggested that PTCA induces inflammatory responses in the coronary circulation. ${ }^{10-14}$

In the present study we investigated changes in concentrations of several cytokines that are supposed to be involved in cascade mechanisms after vascular injury: IL-6, a multifunctional cytokine playing a central role in inflammation and tissue injury; platelet derived growth factor (PDGF), a potent mitogen for vascular smooth muscle cells; macrophage chemoattractant protein 1 (MCP-1), a cyto- kine that induces migration and adhesion of macrophages to endothelial cells; and macrophage colony stimulating factor (M-CSF), which triggers production of various substances from macrophages. We also examined the clinical significance of these cytokines in late restenosis of dilated coronary arteries. As the role of inflammation in patients undergoing PTCA might differ between patients with stable and unstable angina, we excluded those with unstable angina from the present study.

\section{Methods}

PATIENTS

We studied 32 patients (27 male and five female, mean (SD) age 57.4 (9.6) years, range 39 to 76 years) with documented myocardial ischaemia who underwent elective PTCA. PTCA was performed on isolated significant stenotic lesions of the left coronary artery (28 left anterior descending and four left circumflex artery lesions). All patients gave informed consent. Patients were divided into three groups according to the PTCA method used: a plain old balloon angioplasty (POBA) group ( $n=10$ patients), a percutaneous transluminal rotational coronary atherectomy (PTRA) group $(n=10)$, and a stent group $(n=12)$.

Twenty seven patients had stable effort angina and five had a previous myocardial infarction with a positive exercise stress test. The mean (SEM) ejection fraction analysed by left ventriculography was $68(2) \%$, ranging from
Correspondence to: email: uikeda@jichi.ac.jp

Accepted 15 March 2000 
$45 \%$ to $84 \%$. Patients with decompensated heart failure, renal failure, hepatic failure, or contraindication for cardiac catheterisation were excluded.

All patients received $81 \mathrm{mg}$ of aspirin, calcium channel blockers ( $40 \mathrm{mg}$ nifedipine or $90 \mathrm{mg}$ diltiazem), and $40 \mathrm{mg}$ of isosorbide dinitrate before PTCA. Patients expected to undergo coronary stent implantation were given $81 \mathrm{mg}$ of aspirin and $200 \mathrm{mg}$ of ticlopidine.

Seven age matched control subjects without coronary artery stenosis who underwent diagnostic coronary angiography were used as controls.

\section{ANGIOPLASTY PROCEDURE}

Under 1\% lignocaine (lidocaine) hydrochloride local anaesthesia, we cannulated a femoral artery with a 7 French sheath and a femoral vein with a 6 French sheath. Selective coronary angiography was performed and the most accurate evaluation of the stenotic lesion made. A non-ionic contrast medium (ioxaglate) was used in all patients. Heparin (5000 units) was given intravenously before PTCA and continued for 24 hours after PTCA to increase the activated partial thromboplastin time by 1.5 to 2 times the normal control values. A 0.014 inch guidewire was used in POBA and stent implantation and a 0.009 inch guidewire in PTRA to cross the stenotic coronary lesion. PTCA was performed using a monorail balloon catheter system. The number of inflations, the inflation pressure, and the Rotablator setting (Scimed/Boston Scientific, Maple Grove, Minnesota, USA) were decided by the operator. In stent implantation, one stent was implanted in each patient and this was sufficient to cover the stenotic lesions (seven Multi Link stents, Advanced Cardiovascular System Inc, Santa Clara, California, USA; two Palmatz-Shatz stents, Johnson and Johnson Interventional Systems, Waren, New Jersey, USA; and three Wiktor stents, Medtronic Interventional Vascular, Kerkrade, Netherlands). In patients who had stent implantation, angioplasty was performed for pre- and postdilatation. In patients having PTRA, postdilatation of the ablated lesion was accomplished with an inflation pressure of $1-3 \mathrm{~atm}$.

Follow up coronary angiography was done six months after PTCA. If patients had recurrent anginal attacks, coronary angiography was performed before the six month follow up.

\section{BLOOD COLLECTION}

Venous blood samples were obtained by gentle syringe suction through a 5 French catheter from the coronary sinus before and immediately after angioplasty. The initial $3 \mathrm{ml}$ were discarded, as were samples that were not obtained smoothly. We also took blood samples from the femoral artery before and after PTCA in eight patients. In the seven control subjects, samples were obtained through the catheter placed in the coronary sinus before and after diagnostic coronary angiography.
MEASUREMENT OF CYTOKINES

IL-6 concentrations were measured by chemiluminescent enzyme immunoassay kits (Fuji Rebio Co, Tokyo, Japan). PDGF-AB, MCP-1, and M-CSF concentrations were measured by specific enzyme linked immunosorbent assay (ELISA) kits (PDGF-AB: Amersham International, Buckinghamshire, UK; M-CSF, MCP-1: R\&D systems, Minneapolis, Minneapolis, USA). The lower limits of detection of IL-6, PDGF-AB, MCP-1, and M-CSF were $0.1,31.3,31.3$, and $31.3 \mathrm{pg} / \mathrm{ml}$, respectively.

\section{QUANTITATIVE CORONARY ANGIOGRAPHY} ANALYSIS

We used a computer based system for quantitative coronary angiography analysis (MEDIS, Leiden, Netherlands). The measurement of minimum lumen diameter was performed by a single investigator who was unaware of the study results. Late loss index was calculated as late loss/acute gain. Late restenosis was defined $^{15}$ as a loss of more than $50 \%$ of the initial gain achieved or an absolute decrease of at least $20 \%$ of the total lumen diameter at follow up angiography.

\section{STATISTICAL ANALYSIS}

Data are expressed as mean (SEM). Comparisons between the two groups were analysed using the unpaired Student's $t$ test. Differences in the distribution of variables were analysed by Fisher's exact test. Serial changes in variables were evaluated by repeated measure two way analysis of variance (ANOVA) followed by Scheffé's test. Probability values of $p<0.05$ were considered significant.

\section{Results}

RESULTS OF ANGIOPLASTY

Successful coronary dilatation was achieved in all patients (from $0.41(0.09)$ to $2.69(0.10)$ $\mathrm{mm}, \mathrm{p}<0.001)$. There was no acute coronary occlusion in any patient. No major coronary dissection was observed in patients receiving POBA. None of the patients had plasma creatine phosphokinase $(\mathrm{CK})$ concentrations above the upper normal value (70 IU/1). Table 1 summarises patient and lesion characteristics and procedural variables in the three groups. There were no significant differences in the distribution of age, sex, body mass index, lesions receiving PTCA, or American College of Cardiology/American Heart Association (ACC/AHA) lesion type. There were no differences in the distribution of coronary risk factors except hyperlipidaemia. PTCA caused an increase in minimum lumen diameter and a decrease in per cent stenosis of the coronary artery in all cases $(p<0.001)$. Mean minimum lumen diameter after PTCA in the stent group was greater than in the POBA or the PTRA group $(\mathrm{p}<0.05)$.

CHANGES IN CYTOKINE EXPRESSION IL-6 concentrations in the coronary sinus increased after PTCA $(p<0.001$; table 2$)$. There were no differences in the changes in IL-6 concentrations among the three groups. No significant change in IL-6 concentrations was 
Table 1 Patient characteristics

\begin{tabular}{|c|c|c|c|}
\hline & $P O B A$ & PTRA & Stent \\
\hline Number of patients & 10 & 10 & 12 \\
\hline Age (years) (mean (SD)) & $59.0(10.3)$ & $59.3(7.3)$ & $54.6(10.6)$ \\
\hline Male/female & $10 / 0$ & $8 / 2$ & $9 / 3$ \\
\hline Body mass index $\left(\mathrm{kg} / \mathrm{m}^{2}\right)$ & $24.0(1.2)$ & $24.5(0.7)$ & $26.5(1.1)$ \\
\hline Hypertension & 7 & 7 & 10 \\
\hline Hyperlipidaemia & 5 & 1 & 10 \\
\hline Diabetes mellitus & 5 & 5 & 4 \\
\hline Current smoker & 8 & 6 & 6 \\
\hline Family history of IHD & 3 & 1 & 4 \\
\hline AHA lesion (LAD/LCX) & $(7 / 3)$ & $(9 / 1)$ & $(12 / 0)$ \\
\hline ACC/AHA lesion type (type A/B/C) & $(1 / 8 / 1)$ & $(1 / 3 / 6)$ & $(0 / 9 / 3)$ \\
\hline Final balloon size $(\mathrm{mm})$ & $3.15(0.13)$ & $3.00(0.12)$ & $3.23(0.10)$ \\
\hline Maximum inflation pressure (atm) & $10.7(0.9)$ & $2.2(0.9)^{\star}$ & $12.8(0.5)$ \\
\hline Duration of inflation (s) & $244(26) \dagger$ & $140(19)$ & $211(19)$ \\
\hline \multicolumn{4}{|l|}{ Before angioplasty } \\
\hline $\operatorname{MLD}(\mathrm{mm})$ & $0.55(0.21)$ & $0.34(0.13)$ & $0.35(0.13)$ \\
\hline Per cent stenosis & $80.7(6.0)$ & $87.8(4.0)$ & $88.8(4.1)$ \\
\hline \multicolumn{4}{|l|}{ After angioplasty } \\
\hline $\operatorname{MLD}(\mathrm{mm})$ & $2.51(0.16) \ddagger$ & $2.44(0.16) \ddagger$ & $3.05(0.14) \ddagger 5$ \\
\hline Per cent stenosis & $6.6(1.1) \ddagger$ & $7.8(2.3) \ddagger$ & $-1.0(1.2) \ddagger$ \\
\hline Maximum CK value after PTCA (IU/l) & $26(3)$ & $25(4)$ & $22(5)$ \\
\hline
\end{tabular}

Data are mean (SEM) or $\mathrm{n}$ unless otherwise specified.

${ }^{\star} \mathrm{p}<0.001 v$ patients with POBA and stent implantation; $t \mathrm{p}<0.05 v$ patients with PTRA; $\neq \mathrm{p}<0.001 v$ MLD and \% stenosis before angioplasty; $\widehat{s p}<0.05, \Phi p<0.001 v$ MLD and \% stenosis in patients with POBA and PTRA.

ACC/AHA, American College of Cardiology/American Heart Association; CK, creatine kinase; IHD, ischaemic heart disease; LAD, left anterior descending coronary artery; LCX, left circumflex coronary artery; MLD, maximum lumen diameter; POBA, plain old balloon angioplasty; PTCA, percutaneous transluminal coronary angioplasty; PTRA, percutaneous transluminal rotational coronary atherectomy.

Table 2 Changes in IL-6 concentrations ( $\mathrm{pg} / \mathrm{ml})$ induced by PTCA

\begin{tabular}{lll}
\hline & Before PTCA & After PTCA \\
\hline Whole cohort & $2.53(0.36)$ & $3.58(0.50)^{\star}$ \\
Change $(x$-fold $)$ & & $1.56(0.12)$ \\
POBA & $2.14(0.35)$ & $2.69(0.33)^{\star}$ \\
Change $(x$-fold) & & $1.34(0.11)$ \\
PTRA & $2.59(0.66)$ & $4.28(0.97)^{\star}$ \\
Change $(x$-fold) & & $1.82(0.21)$ \\
Stent implantation & $2.82(0.78)$ & $3.76(1.04)^{\star}$ \\
Change $(x$-fold) & & $1.52(0.20)$ \\
\hline
\end{tabular}

Values are mean (SEM).

${ }^{\star} \mathrm{p}<0.001 v$ before PTCA.

found in the femoral arterial blood after PTCA (from $1.65(0.06)$ to $1.83(0.14) \mathrm{pg} / \mathrm{ml})$, nor in coronary sinus blood from the control subjects after diagnostic coronary angiography (from $1.99(0.27)$ to $2.17(0.27) \mathrm{pg} / \mathrm{ml})$.

There were no changes in MCP-1 and M-CSF concentrations after PTCA (MCP-1: from 371 (42) to 356 (34) pg/ml; M-CSF: from $674(85)$ to $693(92) \mathrm{pg} / \mathrm{ml})$. We were only able to measure PDGF concentrations before and after PTCA in five patients because in the other patients the values were beneath the lower limit of detection. In the five patients in whom measurements could be made, no significant change was observed after PTCA (from 133 (54) to $161(58) \mathrm{pg} / \mathrm{ml}$ ).

\section{CORRELATIONS BETWEEN CYTOKINE}

CONCENTRATIONS AND LATE RESTENOSIS

We performed follow up coronary angiography in 28 patients. In these patients successful dilatation of the stenotic coronary artery was achieved (from $0.37(0.07)$ to $2.69(0.11) \mathrm{mm}$, $\mathrm{p}<0.001)$. Mean values of acute gain and late loss were $2.22(0.13)$ and $0.99(0.20) \mathrm{mm}$, respectively. Mean late loss index was 48.9 (7.1)\%. As shown in fig $1 \mathrm{~A}$, a significant positive correlation was observed between changes in IL-6 concentrations and late loss index after PTCA $(r=0.73, \mathrm{p}<0.001)$. On the other hand, there was no significant correlation

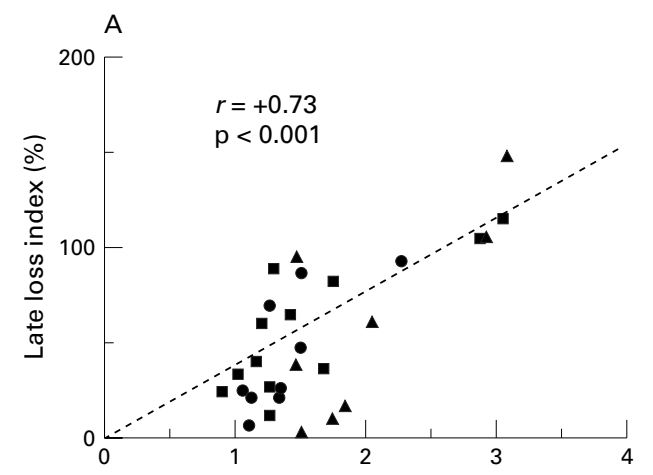

Fold change in IL-6 concentrations after PTCA

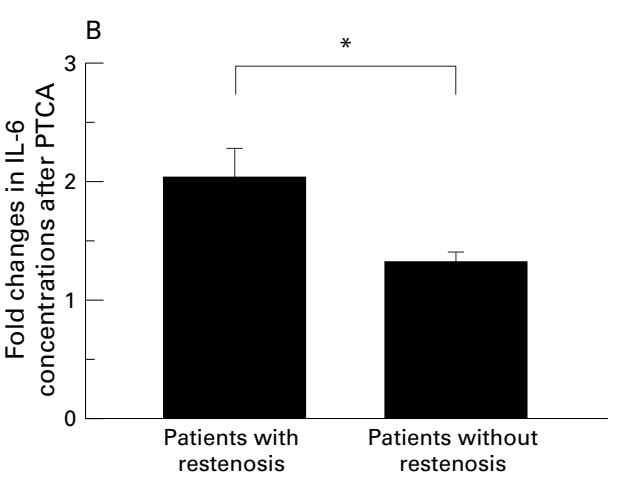

Figure 1 Correlation between changes in IL-6 concentrations in the coronary sinus induced by PTCA and late restenosis. (A) A significant positive correlation was found between relative changes in IL-6 concentrations immediately after PTCA and late loss index six months after PTCA $(r=0.73, p<0.001)$. Circles, data from POBA (plain old balloon angioplasty) group; triangles, PTRA (percutaneous transluminal rotational coronary atherectomy) group; squares, stent group. (B) Patients were divided into two groups according to late restenosis. Patients with restenosis $(n=11)$ showed significantly higher IL-6 concentrations after PTCA than those without restenosis (n $=17)$. Data are means, error bars $=S E M .{ }^{\star} p<0.001$.

between changes in concentrations of MCP-1 or M-CSF and late loss index (data not shown). We could not evaluate the relation between changes in PDGF concentrations and 
late restenosis because of the small number of subjects.

We divided 28 patients into two groups according to the late restenosis criteria. ${ }^{15}$ Eleven showed late restenosis and 17 showed no restenosis (restenosis rate $39.2 \%$ ). As shown in fig $1 \mathrm{~B}$, patients with late restenosis had greater increases in IL-6 concentrations in the coronary sinus blood after PTCA than those without late restenosis $(2.03(0.23) v$ $1.31(0.07) \mathrm{pg} / \mathrm{ml}, \mathrm{p}<0.001)$.

\section{Discussion}

Vascular injury induced by PTCA may upregulate local cytokine expression in dilated coronary arteries and lead to inflammatory responses after PTCA. ${ }^{10-14}$ In our present study, we investigated changes in IL-6, PDGF, MCP-1, and $\mathrm{M}-\mathrm{CSF}$ in the coronary circulation before and after PTCA. We hypothesised that cytokines released from activated macrophages and from the cells forming the vascular wall may play an important role in late restenosis after PTCA by triggering upregulation of vasoactive substances and growth factors. We found significant increases in plasma IL-6 concentrations in the coronary circulation immediately after PTCA, but no increase in MCP-1, M-CSF, or PDGF. It is likely that the increase in IL-6 after PTCA was caused by its release from activated macrophages, lymphocytes, vascular walls, and atherosclerotic lesions, because it takes several hours for the initiation of de novo synthesis. Mechanical stretch, vascular injury, and hypoxia may all induce IL-6 release in the coronary circulation after PTCA. Previous studies, including our own, have shown that human atherosclerotic lesions express substantial amounts of IL-6 mRNA and protein. ${ }^{16}{ }^{17}$

It is possible that changes in cytokine concentrations in the coronary circulation are dependent on the coronary intervention method used. Rotational atherectomy totally resects the atheromatous plaque and the surface endothelium, while stent implantation involves placing a foreign body in the vascular wall. Our statistical analysis showed that the type of intervention did not affect the changes in IL-6 concentrations after PTCA. Although the difference was not significant, the mean change in IL-6 after PTRA was slightly greater than in the other groups. More than half the patients in the PTRA group had irregular heavily calcified lesions on angiography. These complex atherosclerotic lesions may contain more IL- 6 than simple lesions. It is also possible that disruption of platelets and immune cells during high speed rotational atherectomy caused a greater increase in the IL- 6 release.

Pietersma and colleagues found that IL- $1 \beta$ concentrations in the supernatant of cultured monocytes from patients who underwent PTCA were correlated with restenosis of dilated coronary arteries. ${ }^{18}$ Inoue and colleagues found that serum concentrations of soluble intercellular adhesion molecule- 1 were positively correlated with late lumen loss. ${ }^{10}$ Montalescot and associates showed that circulating fibrinogen could be a prognostic marker of restenosis after PTCA. ${ }^{19}$ We found a signifi- cant correlation between changes in IL- 6 concentrations in the coronary circulation after PTCA and the degree of angiographic restenosis. IL-6 is a multifunctional cytokine regulating humoral and cellular responses and it plays a central role in inflammation and tissue injury. It has been reported that IL- 6 is a major inducer of acute phase proteins and other cytokines and growth factors. ${ }^{20}$ IL-6 itself is also said to have procoagulant properties ${ }^{21-23}$ and mitogenic activity for smooth muscle cells. ${ }^{24}$ Thus it may be one of the factors that trigger cascade responses of various substances after PTCA, and an early increase in IL-6 may play a critical role in late restenosis by inducing other cytokines, growth factors, and mitogens. Interestingly, Liuzzo and colleagues reported that there is a group of patients with unstable angina who have an enhanced inflammatory response even to a small stimulus such as diagnostic coronary angiography. ${ }^{14}$ They speculated that this hyperresponsiveness is involved in acute thrombotic complications. Hyperresponsiveness of IL- 6 expression might be an indicator of the degree of inflammation in atheromatous lesions, and such vulnerable lesions are prone to be complicated by acute thrombosis and late restenosis.

We did not find significant increases in MCP-1, M-CSF, or PDGF in the coronary circulation after PTCA. Filonzi and colleagues reported that IL-1, tumour necrosis factor $\alpha$ $(\mathrm{TNF} \alpha)$, and $\gamma$ interferon increased M-CSF mRNA expression in cultured human aortic smooth muscle cells after six hours. ${ }^{25}$ Kranzhöfer and colleagues reported that $\alpha$ thrombin stimulates MCP-1 mRNA expression in vascular smooth muscle cells after six hours of exposure. ${ }^{1}$ Jordan and colleagues also found that IL- $1 \alpha$ and TNF $\alpha$ enhanced MCP-1 mRNA expression in vascular smooth muscle cells, with a peak at 48 hours poststimulation. ${ }^{2}$ Although concentrations of MCP-1 and M-CSF did not change immediately after PTCA, expression of these cytokines may increase several hours after PTCA in response to increased production of thrombin and proinflammatory cytokines. Previously, Caplice and associates reported transient increases in PDGF in the coronary circulation in patients who underwent directional coronary atherectomy. ${ }^{26}$ However, we did not find significant changes in PDGF concentrations in our study. This discrepancy might reflect the different intervention methods used.

LIMITATIONS OF THE STUDY

We did not include any patients with unstable angina, and our findings thus refer only to patients with stable angina. In addition, we only describe the changes in these cytokines that occurred immediately after PTCA. Further study is necessary to investigate the expression of cytokines serially in the hours following PTCA, and to determine the possible clinical significance of cytokine production for late restenosis in dilated vessels. The number of patients was relatively small, and investigations in a larger group of patients would be desirable. 
CONCLUSIONS

PTCA induces a significant increase in IL-6 in the coronary circulation. Increased IL-6 expression may trigger subsequent inflammation and play a critical role in late restenosis of dilated coronary arteries after PTCA.

1 Kranzhöfer R, Clinton SK, Ishii K, et al. Thrombin potentially stimulates cytokine production in human vascular smooth muscle cells but not in mononuclear phagocytes. Circ Res 1996;79:286-94.

2 Jordan NJ, Watson ML, Williams RJ, et al. Chemokine production by human vascular smooth muscle cells; modulation by IL-13. Br ₹ Pharmacol 1997;122:749-57.

3 Mitsumata M, Fishel RS, Nerem RM, et al. Fluid shear stress stimulates platelet-derived growth factor expression in endothelial cells. Am $\mathcal{F}$ Physiol 1993;265:H3-8.

4 Ikeda U, Ohkawa F, Seino Y, et al. Serum interleukin 6 levels become elevated in acute myocardial infarction. $f \mathrm{Mol} \mathrm{Cell}$ Cardiol 1992;24:579-84.

5 Kukielka GL, Smith W, LaRosa GJ, et al. Interleukin-8 gene induction in the myocardium after ischemia and reperfusion in vivo. F Clin Invest 1995;95:89-103.

6 Neumann FJ, Ott I, Gawaz M, et al. Cardiac release of Neumann FJ, Ott I, Gawaz M, et al. Cardiac release of cytokines and inflammatory responses

7 Biasucci LM, Vitelli AA, Liuzzo G, et al. Elevated levels of interleukin-6 in unstable angina. Circulation 1996;94:874-7.

8 Marx N, Neuman FJ, Ott I, et al. Induction of cytokine expression in leukocytes in acute myocardial infarction. $f$ Am Coll Cardiol 1997;30:165-70.

9 Libby P, Schwartz D, Brogi E, et al. A cascade model for restenosis. a special case of atherosclerosis progression. Circulation 1992;86(suppl III):III-47-52.

10 Inoue T, Sakai Y, Hoshi K, et al. Lower expression of neutrophil adhesion molecule indicates less vessel wall injury and might explain lower restenosis rate after cutting balloon angioplasty. Circulation 1998;97:2511-18.

11 Kowalski J, Kosmider M, Pawlicki L, et al. Complement activates neutrophils during PTCA procedure in patients activates neutrophils during PTCA procedure in patients with 40 .

12 Serrano CV, Ramires JAF, Venturinelli M, et al. Coronary angioplasty results in leukocyte and platelet activation with adhesion molecule expression. F Am Coll Cardiol 1997;29: 1276-83.
13 Blum A, Sclarovsky S, Shohat B. T lymphocyte activation in stable angina pectoris and after percutaneous transluminal stable angina pectoris and after percutaneous tra
coronary angioplasty. Circulation 1995;91:20-2.

14 Liuzzo G, Buffon A, Biasucci LM, et al. Enhanced inflammatory response to coronary angioplasty in patients with severe unstable angina. Circulation 1998;98:2370-6.

15 Gimple LW, Herrmann HC, Winnford M, et al, for the Ardeparin and Restenosis Study Group. Usefulness of subcutaneous low molecular weight heaprin (Ardeparin) for reduction of restenosis after percutaneous transluminal coronary angioplasty. Am $\mathcal{F}$ Cardiol 1999; 83:1524-9.

16 Rue HG, Vlaicu R, Niculescu F. Interleukin-6 and interleukin-8 protein and gene expression in human arterial atherosclerotic wall. Atherosclerosis 1996;127:263-71.

17 Seino Y, Ikeda U, Ikeda M, et al. Interleukin 6 gene transcripts are expressed in human atherosclerotic lesions. transcripts are expresse
Cytokine 1994;6:87-91.

18 Pietersma A, Kofflard M, de Wit EA, et al. Late lumen loss after coronary angioplasty is associated with the activation status of circulating phagocytes before treatment. Circulation 1995;91:1320-5.

19 Montalescot G, Ankri A, Vicaut E, et al. Fibrinogen after coronary angioplasty as a risk factor for restenosis. Circulation 1995;92:31-8.

20 Ganter U, Arcone R, Toniatti C, et al. Dual control of $\mathrm{C}$-reactive protein gene expression by interleukin-1 and interleukin-6. EMBO ₹ 1989;8:3773-9.

21 Mestries JC, Kruithof EKO, Gascon MP, et al. In vivo modulation of coagulation and fibrinolysis by recombinant glycosilated human interleukin-6 in baboons. Eur Cytokine Netw 1994:5:275-81.

22 van der Poll T, Levi M, Hack CE, et al. Elimination of interleukin 6 attenuates coagulation activation in experimental endotoxemia in chimpanzees. F Exp Med 1994;179:1253-9.

23 Stouthard JML, Levi M, Hack CE, et al. Interleukin-6 stimulates coagulation, not fibrinolysis, in humans. Thromb Haemost 1996;94:874-7.

24 Ikeda U, Ikeda $M$, Oohara $T$, et al. Interleukin-6 stimulates the growth of vascular cells in a PDGF dependent manner. Am F Physiol 1991;260:H1713-17.

25 Filonzi EL, Zoellner H, Stanton H, et al. Cytokine regulation of granulocyte-macrophage colony stimulating factor and macrophage colony stimulating factor production in human arterial smooth muscle cells. Atherosclerosis 1993; 99:241-52.

26 Caplice NM, Aroney CN, Bett JHN, et al. Growth factors released into the coronary circulation after vascular injury promote proliferation of human vascular smooth muscle cells in culture. F Am Coll Cardiol 1997;29:1536-41. 